\title{
Le dessin-entretien : un outil de collecte de données innovateur et approprié auprès des communautés culturelles et linguistiques minoritaires
}

\author{
Constance Lavoie, Ph.D.
}

\author{
Université du Québec à Chicoutimi
}

Jo-Anni Joncas, Doctorante

Université du Québec à Chicoutimi

\begin{abstract}
Résumé
L'objectif de cet article est de présenter un outil de collecte de données qualitatives pour les chercheurs travaillant auprès de communautés linguistiques et culturelles minoritaires. Il s'agit du dessin-entretien. Cet outil, basé sur le dessin et le groupe de discussion, est utilisé dans un processus cyclique qui permet un temps de réflexion personnelle et d'échanges entre les participants. Ce processus se déroule en quatre étapes : le dessin individuel, la présentation individuelle, le dessin collectif et le groupe de discussion. Le dessin-entretien a été développé et expérimenté au cours de deux recherches, d'abord auprès d'enseignants Hmongs au Vietnam puis auprès d'universitaires membres des Premières Nations du Québec. Les résultats des études indiquent que l'accès à diverses modalités de collecte facilite les échanges entre les participants et augmentent la triangulation des informations.

Mots clés

MinORITÉS ETHNOCULTURELLES, OUTIL DE COLLECTE DE DONNÉES, RECHERCHE QUALITATIVE, DESSIN-ENTRETIEN
\end{abstract}

Note des auteures : Les auteures remercient le Fonds de recherche du Québec - Société et culture (FQRSC) et le Conseil de recherches en sciences humaines du Canada (CRSH), car ces données proviennent de projets de recherches subventionnés par ces organismes. De plus, elles remercient les participants à ces recherches pour leur apport incomparable.

RECHERCHES QUALITATIVES - Vol. 34(1), pp. 97-121.

LA RECHERCHE QUALITATIVE AUJOURD'HUI : RÉFLEXIONS ET PRATIQUES

ISSN 1715-8702 - http://www.recherche-qualitative.qc.ca/revue/

(C) 2015 Association pour la recherche qualitative 


\section{Introduction}

Cet article vise à présenter l'outil qu'est le dessin-entretien. Cet outil de collecte de données a été élaboré afin de répondre aux lacunes méthodologiques liées aux recherches intégrant des communautés culturelles et linguistiques minoritaires. Le chercheur travaillant auprès de ces communautés est souvent confronté à des limites méthodologiques relatives à des incompréhensions linguistique, langagière et culturelle (Haig-Brown, 2010). Nombreux sont les questionnements relatifs à l'interprétation des silences, des gestes, des expressions, des inférences ou des métaphores lors des collectes de données. Le chercheur se trouve devant un dilemme. D'un côté, il peut omettre certaines informations fort révélatrices ou, de l'autre, il peut tenter de les interpréter sans avoir la certitude de la justesse de son jugement. Par ailleurs, le choix d'outils cohérents pour étudier des problématiques liées aux minorités ethnoculturelles, aux groupes marginalisés ou aux populations autochtones est important afin d'être en mesure de bien saisir l'objet d'étude du point de vue des acteurs concernés (Smith, 2003). Au regard de ces constatations méthodologiques et pour se sortir du dilemme, Lavoie (2011) a développé un outil, le dessin-entretien, en se basant sur celui du photovoice de Wang et Burris (1997) afin de respecter le style de communication des minorités ethnoculturelles pour qui le silence, la narration en groupe et les représentations visuelles ont une grande importance. En effet, par sa sensibilité culturelle et sa multimodalité, le dessin-entretien ajoute à l'entretien de groupe un aspect visuel et un espace collectif d'interprétation et de triangulation des données. Ainsi, l'outil du dessin-entretien facilite la recherche auprès de minorités ethnoculturelles, car il prend appui sur la spécificité des contextes et des modes de communication (Denzin, Lincoln, \& Smith, 2008; Restoule, Archibald, Lester-Smith, Parent, \& Smilie, 2010; Smith, 2003; Stocek \& Mark, 2009; Styres, Zinga, Bennett, \& Bomberry, 2010; Wilson, 2003).

\section{Cadre théorique}

Lorsqu'il est question d'entrer en relation avec des minorités ethnoculturelles, il est primordial de prendre en considération leurs particularités et leur réalité. Par conséquent, les postures méthodologiques des recherches auprès des peuples autochtones révèlent certains principes à respecter. Il y a d'abord le principe du savoir relationnel. Ce principe conçoit le savoir comme étant partagé et construit dans la relation entre les personnes plutôt que de le concevoir comme étant un objet de connaissance détenu par un expert (Absolon \& Willet, 2004; Battiste, 2008; Smith, 2003; Stocek \& Mark, 2009; Weber-Pillwax, 1999). De cette façon, le savoir et la connaissance se créent dans la relation et le dialogue entre les personnes. Il est alors préférable de 
favoriser de bonnes relations entre tous les participants, incluant le chercheur, lors des différentes étapes de la recherche avec des minorités ethnoculturelles, et particulièrement lors de la collecte de données (Weber-Pillwax, 1999). Martin et Mirraboopa (2003) sont du même avis en avançant qu'il vaut mieux privilégier la voix et les expériences de vie des participants concernés en contexte de recherche avec les peuples autochtones. Les valeurs traditionnelles centrales des cultures autochtones, telles que le respect, la responsabilité, la réciprocité et la révérence, doivent également être intégrées à l'intérieur du processus de recherche (Archibald, 2008). Des auteurs recommandent aussi de placer les participants à l'intérieur d'un cercle, ce qui rappelle la dynamique circulaire autochtone (Foy, 2009; Lavallée, 2009). Styres et al. (2010) ajoutent que le cercle est indispensable pour créer un espace discursif commun entre les participants et pour créer une atmosphère collaborative, dynamique et relationnelle. Enfin, pour parvenir à cette construction commune du savoir, Foy (2009) indique que chaque participant doit avoir une grande écoute, doit partager et doit être ouvert. Les participants doivent être patients, faire preuve de confidentialité quand des expériences de la vie privée sont partagées et participer jusqu'à la fin de la discussion (Foy, 2009). Chaque membre et chaque propos amené ont une importance égale et équitable (Styres et al., 2010). Les principes hiérarchiques sont alors à proscrire de même que les jugements (Absolon \& Willet, 2004; Smith, 2003; Weber-Pillwax, 1999). Il vaut mieux favoriser les principes de l'holisme, de l'interrelation et de la synergie. Le dessin-entretien a été élaboré en considérant tous ces principes et ces valeurs.

Dans un premier temps, le dessin-entretien s'appuie sur le dessin pour produire, collecter et analyser des données dans le but d'atteindre une meilleure compréhension de l'objet étudié. Depuis plusieurs siècles, les méthodes de recherche visuelles sont utilisées dans différentes disciplines telles que la psychologie, la sociologie et l'anthropologie (Knowles \& Cole, 2008). Finley (2008) indique que les recherches utilisant des méthodes artistiques comme le dessin ont le potentiel de faciliter l'investigation auprès des peuples opprimés et rejettent les pratiques de tradition colonialiste ou paternaliste. Elles sont notamment utilisées en contexte de distance langagière entre le chercheur et les participants et visent à faire ressortir les perceptions des participants à travers des moyens non verbaux (Bishop, 2006). En effet, Weber (2008) souligne que les images permettent d'exprimer ce qui peut être difficile de mettre en mots, surtout en contexte de langue seconde. Les dessins sont alors perçus comme des artéfacts qui expriment des significations (Stock, Mares, \& Robinson, 2012). Un artéfact est un type de matériel (par exemple un dessin ou un collage) ou un symbole (par exemple un pictogramme) qui peut devenir un 
moyen de communication (Lavoie \& Benson, 2011; Swain, Kinnear, \& Steinman, 2011). Par exemple, les Hmongs utilisent beaucoup la broderie, Paj Ntaub, pour raconter des légendes et leur passé historique (Collignon, 1994; Rudduck \& Hopkins, 1985; Shea \& Yang, 1995). Utilisée dans de bonnes conditions, la conception d'artéfact, comme le dessin, encourage une certaine transparence et authenticité et demande un processus réflexif de la part des participants (Weber, 2008). Barthes (1981, 1983) ajoute que les dessins évoquent deux significations dont l'une se rapporte à l'aspect apparent, évident et objectif du dessin, et l'autre, plutôt connotative, ne pouvant être expliquée que par l'auteur. Ainsi, les instruments de collecte utilisant le dessin ont le potentiel de saisir en profondeur le sens qu'attribuent certains participants à l'objet d'étude. D'autres recherches ont également utilisé le dessin, notamment en éducation, afin de mieux comprendre l'expérience et le vécu des participants (Bishop, 2006; Fournier \& Côté, 2008; Kendrick \& Jones, 2008; Stock et al., 2012), pour établir des représentations de jeunes (Ehrlen, 2009; Kendrick \& McKay, 2004) et pour faciliter la communication entre des participants et des chercheurs possédant des différences linguistiques (Bernhard, Cummins, Campoy, Ada, Winsler, \& Bleiker, 2006; Prasad, 2010; Salem, 2000).

Dans un second temps, l'outil du dessin-entretien utilise les ancrages théoriques du groupe de discussion. Le groupe de discussion est connu sous plusieurs appellations : l'entretien de groupe focalisé, le groupe focalisé, le focus group, etc. (Leclerc, Bourassa, Picard, \& Courcy, 2011). Malgré les légères distinctions théoriques et les ancrages épistémologiques, le groupe de discussion peut être défini comme «une méthode de collecte de données qualitatives réunissant un nombre restreint de personnes dans une conversation centrée sur un sujet ou un champ d'intérêt défini qui a fait, fait ou fera partie de l'existence des personnes regroupées » (Leclerc et al., 2011, p. 146). Le groupe de discussion est un outil polyvalent permettant de réduire l'écart entre les données probantes et la pratique dans un contexte d'interaction sociale et de coconstruction de sens (Morrissette, 2011). La pertinence des groupes de discussion menés auprès de groupes culturels et linguistiques minoritaires a été soulevée dans plusieurs études (Calderon, Baker, \& Wolf, 2000; Halcomb, Gholizadeh, DiGiacomo, Phillips, \& Davidson, 2007; Överlien, Aronsson, \& Hydén, 2005). Récemment, l'étude de Birch et Pétry (2011) réaffirmait que

[1]es groupes de discussion permettent de mieux comprendre les groupes linguistiques et culturels minoritaires et les groupes marginalisés ou vulnérables, contribuant ainsi à améliorer les interventions sociales et les services auprès de ces groupes, notamment en cernant mieux leurs besoins (p. 105). 
Bien que soulevé par les études en sciences de la santé, cet avantage est tout aussi valable pour les sciences de l'éducation. En éducation, les groupes de discussion contribuent à la formalisation de connaissances spécifiques et pratiques qui sont essentielles lors d'interventions éducatives adéquates auprès des minorités ethnoculturelles (Baribeau \& Germain, 2010).

Dans une moindre mesure, le dessin-entretien se base également sur le principe de l'approche narrative. L'approche narrative est l'approche traditionnellement utilisée chez les peuples autochtones tant dans la vie de tous les jours que dans l'éducation des enfants (Stock et al., 2012). En effet, la tradition orale est commune à toutes les nations autochtones (McMullen \& Rohrbach, 2003). Grâce à elle, les légendes et les histoires traditionnelles circulent de génération en génération. Ces contes oraux permettent la transmission symbolique et métaphorique des valeurs et des coutumes de la communauté. De cette façon, l'échange par la narration est connu par les peuples autochtones et facilite la prise de parole (Stock et al., 2012).

\section{Cadre méthodologique}

Il convient de spécifier les contextes de recherche dans lesquels le dessin-entretien a été développé et expérimenté. L'élaboration de cet outil résulte de deux recherches ayant été réalisées avec des communautés linguistiques et culturelles minoritaires. D'abord développé auprès d'une communauté Hmong au nord du Vietnam, le dessin-entretien a par la suite été bonifié grâce à une expérimentation avec un groupe d'étudiants universitaires des Premières Nations au Canada.

\section{Contextes}

Dans un premier temps, le dessin-entretien a été développé dans le cadre d'une recherche-action portant sur l'éducation bilingue (langue ethnique et langue vietnamienne) auprès de trois groupes ethniques minoritaires (les Hmongs, les Khmers et les J'rais) (Benson \& Kosonen, 2011). Cette recherche-action était entreprise par le ministère de l'Éducation et de la Formation du Vietnam et supportée par le bureau d'UNICEF ${ }^{1}$ au Vietnam. Parmi l'équipe de chercheurs, l'une d'entre eux (Lavoie, 2008) avait comme mandat de développer un outil de recherche permettant d'approfondir les causes expliquant les faibles taux d'alphabétisation et les piètres résultats scolaires des Hmongs du Vietnam (Kosonen, 2004; UNICEF, MOET ${ }^{2}, \mathrm{UNESCO}^{3}$, \& $\mathrm{UNGEI}^{4}, 2008$ ). Par la suite, elle devait initier les enseignants à cet outil pour qu'ils l'appliquent durant l'année scolaire pour échanger sur les conditions éducatives des minorités ethnoculturelles. L'expérience de recherche d'une des auteures auprès des Hmongs du Vietnam (Lavoie \& Benson, 2011) a orienté le choix vers le dessin plutôt que la photo, car l'utilisation d'un appareil-photo entraîne 
davantage de contraintes situationnelles (coût élevé, vol, perte, connaissances techniques).

La richesse des informations recueillies grâce à l'utilisation du dessinentretien lors de la recherche de Lavoie (2011) auprès de minorités ethnoculturelles du Vietnam a incité Joncas (2013) à choisir cet instrument lors de sa recherche de maîtrise portant sur l'expérience scolaire d'étudiants universitaires des Premières Nations. Ainsi, cette nouvelle utilisation du dessinentretien a permis d'optimiser l'outil et de l'utiliser avec un autre groupe ethnique minoritaire, les membres des Premières Nations du Québec. L'étude tentait de comprendre le sens de l'expérience scolaire d'étudiants des Premières Nations qui ont persévéré au niveau universitaire, ces expériences étant qualifiées d'exceptionnelles et d'atypiques aux dires des statistiques (Ministère de l'Éducation, du Loisir et du Sport, 2009).

\section{Participants}

$\mathrm{Au}$ Vietnam, vingt-cinq enseignants ont participé au dessin-entretien. Ces enseignants travaillaient dans quatre communautés du nord-ouest du Vietnam : Bac Pho, Sa Pa, Sin Cheng et Ta Van Chu. Parmi les participants, il y avait six hommes et dix-neuf femmes, dont douze Hmongs, huit Kinhs (l'ethnie majoritaire du Vietnam), quatre Tays et un Nuong. Étant donné leur grand nombre, les participants vietnamiens étaient divisés en deux groupes. Tous ces enseignants travaillaient dans une école primaire ou préscolaire. Les participants étaient âgés de 19 à 49 ans. Dans le cadre de la seconde expérimentation, sept étudiants de l'Université du Québec à Chicoutimi (UQAC) issus de communautés autochtones ont participé à cette recherche. Il s'agissait de six femmes et d'un homme âgés de 22 à 30 ans.

\section{Déroulement}

Au Vietnam, la collecte de données avec le dessin-entretien s'est déroulée sur quatre jours. La chercheuse s'exprimait en anglais et les participants en vietnamien. Les discussions étaient traduites simultanément par un membre du personnel d'UNICEF. Tous les participants parlaient et écrivaient en vietnamien. L'expérimentation a eu lieu dans une école de la ville de Lao Caï, au Vietnam. Lors de la seconde expérimentation, la collecte de données a eu lieu à l'Université du Québec à Chicoutimi sur une période de deux jours. Les conversations se tenaient principalement en français. À l'occasion, certains participants provenant de la même nation parlaient entre eux dans leur langue maternelle autochtone. Dans ces deux contextes d'expérimentation, le dessinentretien s'est déroulé en deux temps de trois heures. 


\section{Journal de bord}

Lors des deux phases d'expérimentation, un journal de bord a été tenu et celuici a permis de colliger suffisamment d'informations pour développer et améliorer le dessin-entretien. Le journal de bord a principalement servi à noter des données descriptives, méthodologiques et analytiques. Avant, pendant et immédiatement après chaque collecte de données, les chercheuses y ont inscrit des observations, des notes théoriques, des intuitions, des hypothèses, des émotions, des questionnements, des éléments contextuels et des réflexions: " [Participant 1] gigote beaucoup quand il parle de la mort de son frère et des suicides. » (Notes de J. Joncas, Canada, 25 janvier 2012). Ces descriptions détaillées et méthodiques de même que les autres notes théoriques du journal de bord ont permis de conserver le caractère unique des situations.

Ces expérimentations ont permis d'essayer le dessin-entretien auprès de différentes minorités ethnoculturelles. La partie suivante présente les constats obtenus à la suite de ces expérimentations lors des différentes étapes du dessinentretien.

\section{Résultats}

L'outil du dessin-entretien se base sur un processus cyclique et est divisé en quatre étapes. Ces quatre étapes sont fixes, mais elles sont flexibles et adaptables aux différentes situations (voir la Figure 1).

\section{Le dessin individuel}

La première étape consiste à répondre à une consigne initiale du chercheur au moyen d'un dessin. La consigne initiale peut également prendre la forme d'une question. Dans l'expérimentation auprès des Hmongs du Vietnam, la chercheuse a donné la consigne suivante : "Dessine-toi comme enseignant dans une communauté hmong. » Dans l'expérimentation auprès d'étudiants des Premières Nations, la consigne était: «Dessine comment tu te perçois à l'Université. » $\mathrm{Au}$ besoin, le chercheur peut ajouter des indications supplémentaires à sa consigne initiale. Il peut s'adresser à l'ensemble du groupe, à quelques-uns d'entre eux ou à un individu en particulier en cas d'incompréhension ou pour relancer la réflexion après un certain temps. Par exemple, Joncas (2013) a spécifié sa consigne en demandant aux participants de se dessiner à l'Université. Le chercheur précise également que les participants peuvent utiliser des pictogrammes ou des symboles pour représenter des idées qu'ils n'arrivent pas à dessiner. Il est important de rappeler qu'aucune valeur artistique n'est attribuée au dessin, et que l'importance est accordée à la signification: «Peu d'attention est accordée au fait que le dessin est beau ou laid, nous nous attardons plutôt à la signification de l'image." [traduction libre] (Enseignante hmong. Notes C. Lavoie, 


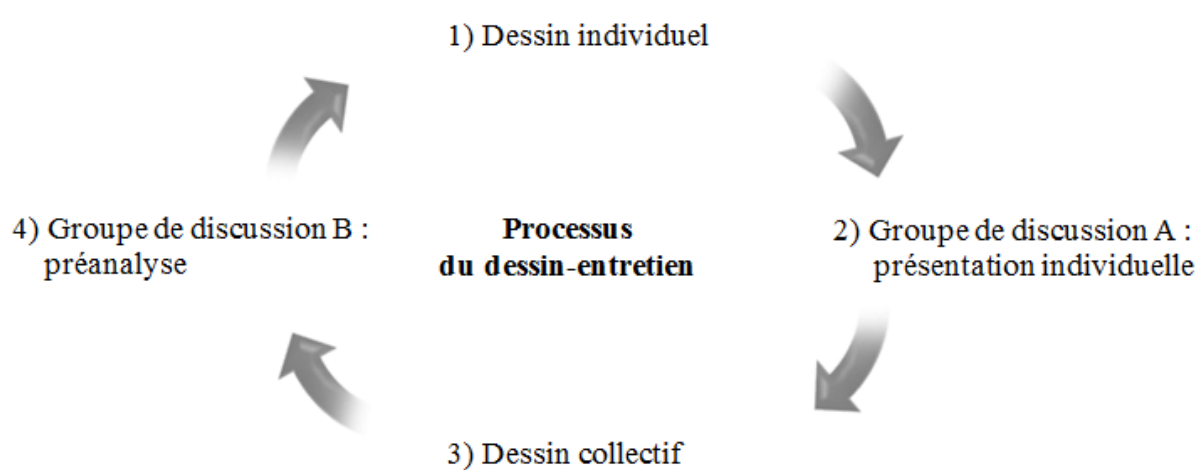

Figure 1. Processus du dessin-entretien.

Vietnam, 30 juillet 2008). Une période de silence et de réflexion suit cette première indication. À la lumière de l'expérimentation auprès des jeunes autochtones du Québec, cette période peut durer dix minutes et même plus. Des participants dessinent leurs idées sans hésitation tandis que d'autres ont besoin d'un temps de réflexion pour visualiser et pour intérioriser leur réponse : «Wow, ça m'étonne de voir tous ces adultes en train de dessiner [...], mais certains ne font rien. Je ne vois pas trop où ça va en venir» (Notes de C. Lavoie, Vietnam, 29 juillet 2008). L'inaction de certains participants peut être déstabilisante pour le chercheur non initié. Toutefois, cette inaction physique ne signifie pas pour autant que le participant n'est pas en train d'approfondir ses idées. La phase du dessin individuel laisse aux participants le temps dont ils ont besoin pour s'exprimer. En effet, grâce au temps de réflexion, les répondants ont dessiné et raconté des passages forts et significatifs de leur expérience scolaire. Par exemple, au moyen de leurs dessins, ils expliquaient les raisons des statistiques élevées d'absentéisme des élèves hmongs. Un enseignant a dessiné un élève sur ses épaules en précisant que les longs déplacements en montagne sont ardus pour les élèves et que les faibles ressources financières des familles entraînent beaucoup d'absences (voir la Figure 2). En même temps qu'ils dessinent, ils réfléchissent et communiquent leurs idées au moyen de leur représentation visuelle. Durant la période du dessin individuel, le chercheur demande aux participants d'ajouter une phrase significative ou une note descriptive à leur dessin pour lui donner voix. Cette note peut consister en des mots clés ou des phrases complètes, et ce, dans la langue de leur choix. 


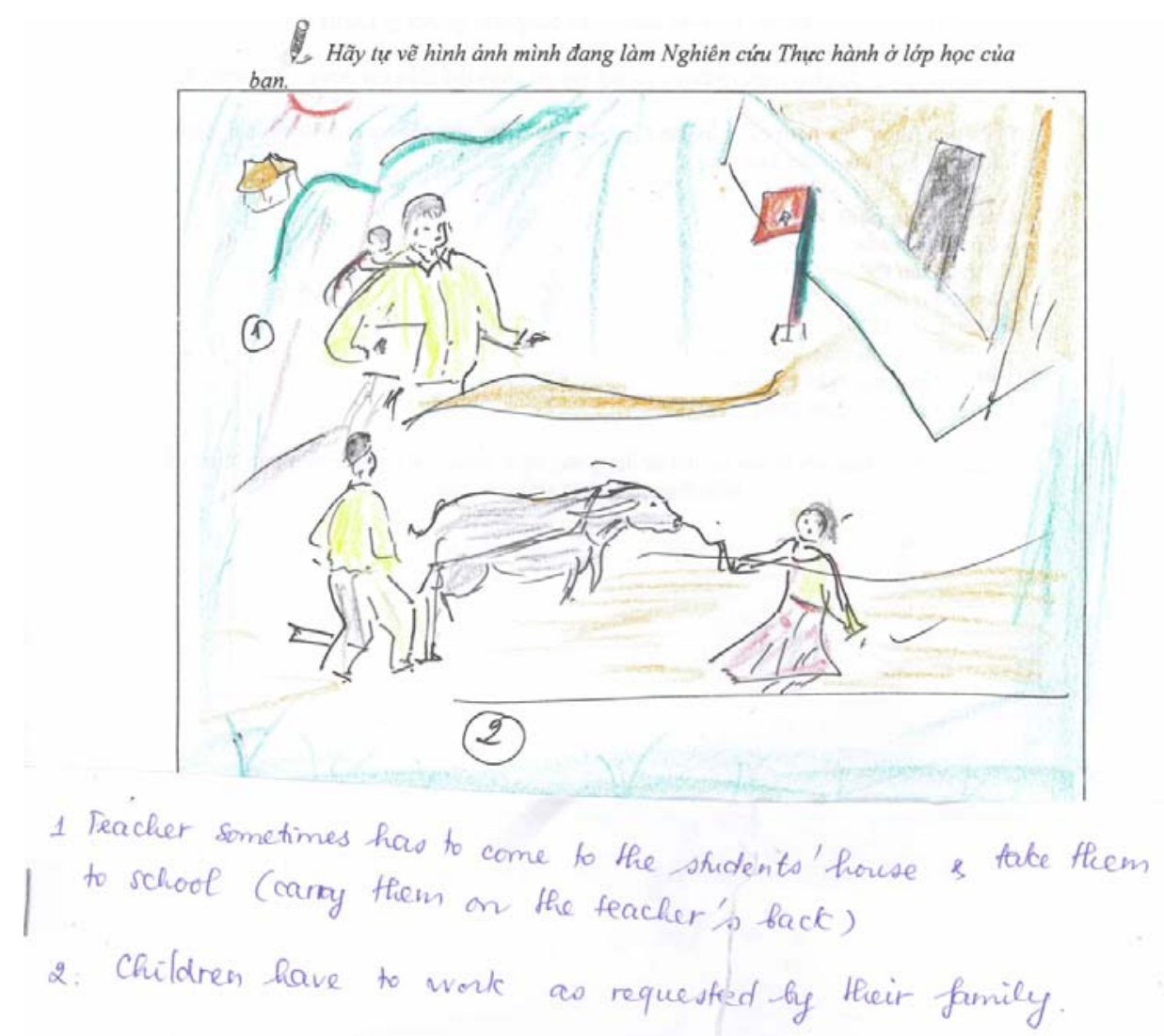

Figure 2. Dessin individuel d'un enseignant hmong avec notes descriptives.

\section{Le groupe de discussion $A$ : la présentation individuelle}

La deuxième étape du dessin-entretien est la présentation individuelle des dessins. Toutes les personnes présentes se placent en cercle. Au fur et à mesure que les participants terminent leur dessin, ils le présentent au groupe. Cette présentation permet entre autres aux participants de se familiariser les uns aux autres : "Globalement, très belle ambiance, ouverture d'esprit, grand respect et je suis surprise à quel point les participants sont ouverts même s'ils ne se connaissent pas entre eux » (Notes de J. Joncas, Canada, 25 janvier 2012). Tout au long de cette étape, le chercheur ou tout autre participant peut interroger le présentateur sur la signification de certains éléments d'un ou des dessins, par exemple : «Pourquoi n'avez-vous pas dessiné vos cours d'eau bleus? » (en faisant référence à de nombreux dessins individuels ayant des rivières brunes) 
(Notes de C. Lavoie, Vietnam, 30 juillet 2008). La réponse donnée par les participants a alors permis de comprendre que le problème soulevé survenait durant une période annuelle d'inondations. Durant ces présentations, les participants ont le choix de l'ordre des thèmes et des éléments qu'ils souhaitent aborder et prioriser. Les données collectées lors de cette étape rejoignent davantage la réalité subjective des répondants. Durant les présentations, l'animateur peut ramener les propos si la discussion s'éloigne du sujet de l'étude. Pendant qu'une personne présente, les autres peuvent arrêter de dessiner pour écouter les présentations ou pour poser des questions, ou ils peuvent continuer de dessiner. Ces interactions, visuelles ou verbales, permettent de compléter leurs idées selon la modalité voulue. Comme l'indiquent Lavoie et Benson (2011),

l'avantage du dessin-entretien est de permettre aux participants de continuer leur dessin tout en parlant ou en écoutant les autres. Au lieu d'interrompre la discussion, certains dessinent leurs idées pour nourrir la discussion ou en signe d'accord avec l'idée amenée par celui qui parle ${ }^{5}$ [traduction libre] (p. 16).

L'une des chercheuses a noté que les participants procédaient naturellement à une thématisation en empilant les dessins, en les classant ou en pointant des similitudes (voir la Figure 3). Par exemple, certains dessinaient des écoles vides pour signifier le manque de ressources gouvernementales qui leur sont accordées (Lavoie, 2011). Ce témoignage visuel a fait consensus de façon silencieuse. Les participants prenaient leur dessin individuel et les empilaient en signe d'accord. Cet accord non verbal semblait beaucoup moins compromettant qu'un accord verbal.

\section{Le dessin collectif}

Durant la troisième étape du dessin collectif, le chercheur demande au groupe de réaliser un dessin collectif ou de choisir un dessin d'un participant pouvant être modifié représentant les idées de tous les membres du groupe. Le groupe doit choisir un animateur, car chaque élément intégré au dessin collectif suit un processus consensuel. Cet animateur coanime avec le chercheur la discussion de groupe. Il a pour rôle de donner le droit de parole, de s'assurer du bon fonctionnement de la discussion de groupe et d'effectuer les modifications décidées collectivement sur le dessin. Le chercheur, quant à lui, alimente la discussion en posant des questions, par exemple. Dans le cas de la recherche au Vietnam, le groupe avait choisi l'enseignante hmong la plus âgée. Dans le cas de la recherche avec des étudiants universitaires des Premières Nations, une participante innue maitrisant aisément le français s'est portée volontaire. Lors de cette étape, le chercheur, tout comme l'animateur, doit reconnaître et tenir 


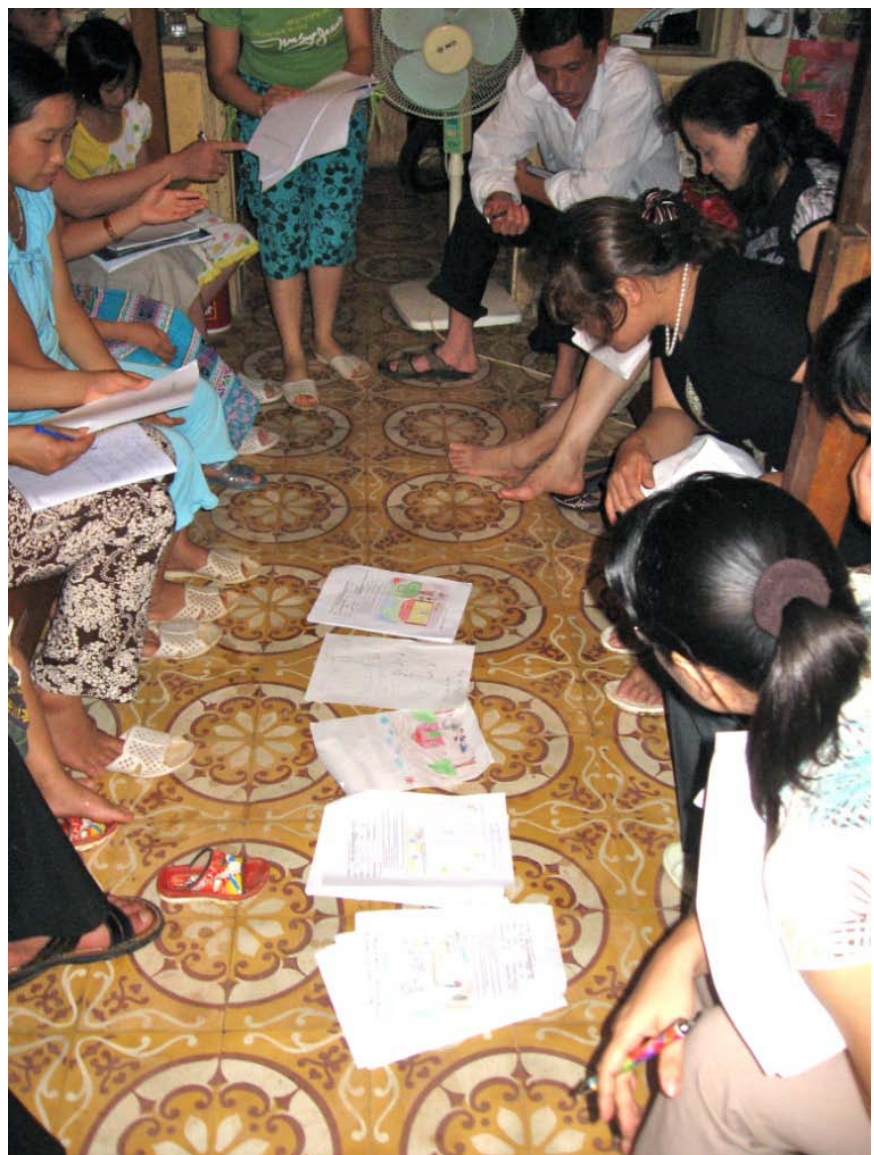

Figure 3. Présentation individuelle et première thématisation (Lavoie, 2008).

compte des éléments de sa subjectivité en essayant d'en diminuer les effets sur la recherche en n'orientant pas le choix des éléments du dessin collectif. Dans ce moment de construction consensuel, un dessin rejoignant l'expérience de tous les participants prend forme. Selon l'expérience des chercheuses, il est peu probable que le groupe choisisse un seul dessin individuel. Par contre, les membres du groupe peuvent choisir des idées des différents participants en découpant les éléments des dessins individuels qui regroupent les idées de plusieurs participants et en les collant sur un grand carton (voir la Figure 4). Le chercheur rappelle que les éléments choisis doivent rejoindre tout le groupe formant ainsi un dessin collectif. En plus des éléments découpés, de nouveaux éléments peuvent être dessinés sur le dessin collectif afin de le compléter. Par 


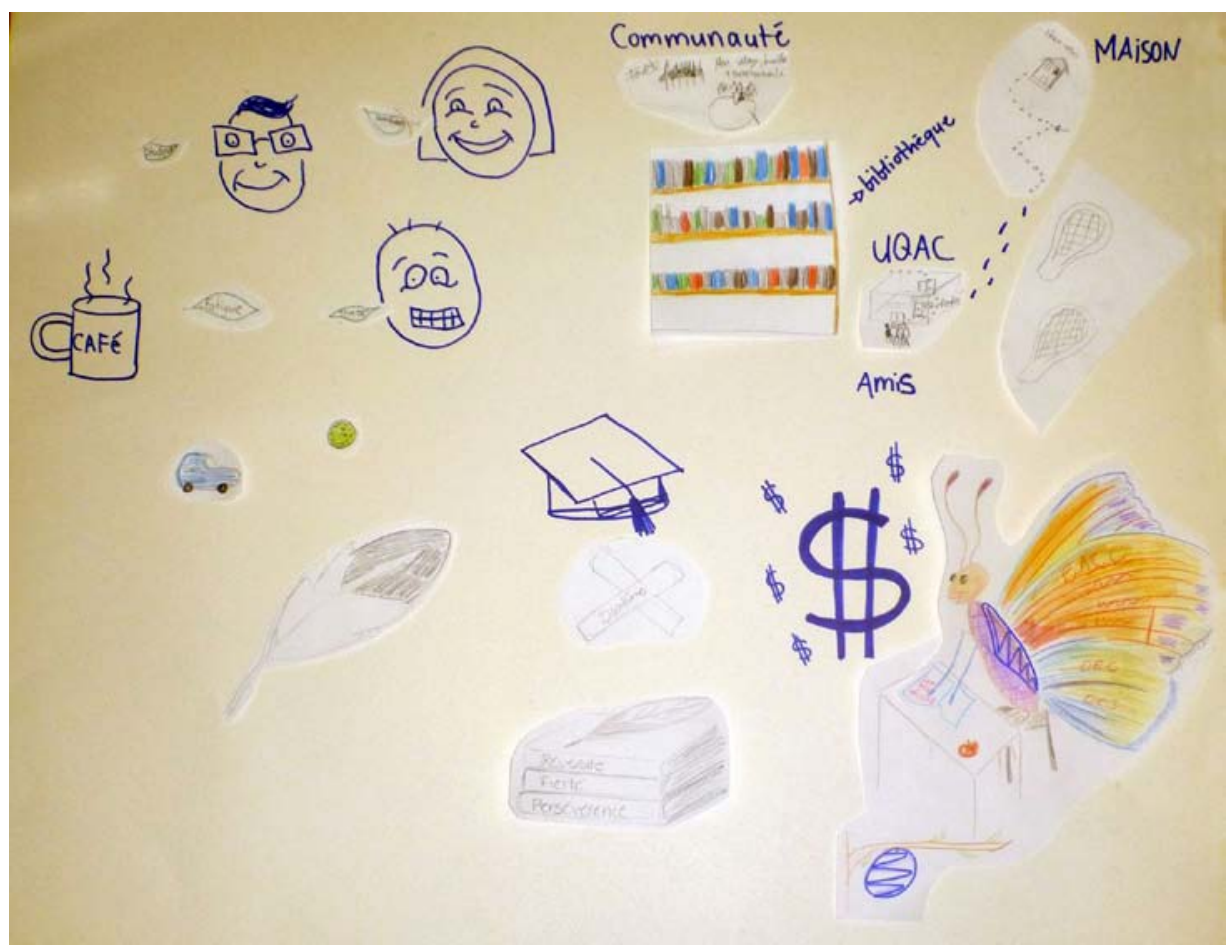

Figure 4. Le dessin collectif: regroupement des éléments choisis par les répondants qui témoignent du sens de leur expérience scolaire à l'Université (Joncas, 2013, p. 97).

conséquent, la mutuelle influence, la coopération et la discussion entre les participants autour de la construction consensuelle du dessin collectif mènent à une coconstruction de l'analyse du sens de l'objet de l'étude. Ce processus négocié de mise en forme soumet les participants à une démarche réflexive comportant des sélections et des hiérarchisations d'éléments plus pertinents que d'autres. Par leur choix consensuel, les éléments figurant sur le dessin collectif sont ainsi liés à chaque participant. Par exemple, la plume figurant sur la Figure 4 du dessin-entretien collectif des étudiants universitaires des Premières Nations a été choisie à l'unanimité par tout le groupe pour figurer sur le dessin collectif.

Il a d'abord été choisi parce qu'il était présent sur plus de la moitié des dessins-entretiens individuels. Également, ce symbole, représentant la culture autochtone et la fierté d'être membre des Premières Nations pour les étudiants 
l'ayant dessiné, rejoignait tous les participants. Ces éléments servent de thèmes de base à l'analyse et à l'interprétation des données.

\section{Le groupe de discussion $B$ : la préanalyse}

Le groupe de discussion est formellement la quatrième étape du dessin-entretien, mais il s'entame durant les discussions entourant l'élaboration du dessin collectif et il se poursuit par la suite. L'entretien peut se dérouler directement à la suite de l'étape précédente ou lors d'une seconde rencontre. Le temps de réflexion entre les deux collectes offre l'avantage au chercheur d'effectuer une préanalyse en cours de transcription des discussions de la première rencontre. Lors de la seconde rencontre, les participants peuvent alors valider la préanalyse du chercheur et l'ajuster au besoin. Cela permet aussi d'éclairer le chercheur à propos de certains sujets ou passages qui lui sont moins clairs. De cette façon, le groupe de discussion permet de creuser les thèmes ressortis, mal compris ou écartés lors du dessin collectif. Durant cette dernière phase, les participants échangent entre eux et harmonisent leurs réflexions individuelles et leurs représentations visuelles avec celles des autres. D'ailleurs, certains éléments peuvent à première vue sembler anodins, comme des raturages ou des petits signes incompréhensibles, mais ils prennent un tout autre sens grâce au groupe de discussion. Le chercheur peut aussi questionner le groupe sur les raisons les ayant amenés à exclure certains éléments du dessin collectif qui lui semblaient importants ou sur les raisons qui les ont poussés à ajouter certains éléments ne figurant sur aucun des dessins individuels, par exemple : « Pourquoi avez-vous ajouté le mot ami? » (voir la Figure 4, élément H) (Notes de J. Joncas, Canada, 25 janvier 2012).

\section{Discussion}

Il est légitime de se poser la question suivante : pourquoi avoir combiné le dessin au groupe de discussion? Les auteures sont enclines à croire que ce choix est congruent aux recherches auprès des groupes minoritaires et marginalisés. Avant de confirmer leur choix méthodologique pour mener leur étude, les auteures avaient d'abord consulté différentes personnes ayant travaillé avec les Hmongs et les peuples autochtones du Canada, dont des chercheurs universitaires et des coopérants d'ONG. Ces experts conseillaient d'opter pour une méthode de recherche visuelle et créatrice avec ces populations, car leurs expériences de recherche leur avaient révélé qu'il est difficile de trouver des participants qui acceptent de témoigner de leur vie personnelle. La recommandation d'utiliser des méthodes visuelles et de groupe pour mener des recherches auprès des peuples minoritaires et marginalisés se retrouve également dans la littérature (Birch \& Pétry, 2011; Kendrick \& McKay, 2004; Prasad, 2010; Stock et al., 2012). En outre, la représentation 
visuelle et la narration font partie des modes de communication des Hmongs (Collignon, 1994; Rudduck \& Hopkins, 1985; Shea \& Yang, 1995) et des peuples autochtones en général (Smith, 2003; Stock et al., 2012).

\section{Comparaison entre le groupe de discussion et le dessin-entretien}

Il est également pertinent de se demander si les données auraient été de qualité équivalente avec seulement des groupes de discussion. En effet, pourquoi ne pas avoir utilisé seulement les groupes de discussion? Une des raisons est que l'entretien et les groupes de discussion sont des outils ayant de lourds passés coloniaux pour certaines populations. Dans le cas des Hmongs, cette crainte de l'étranger s'explique en partie par le fait que durant la guerre du Vietnam (1959-1975), les Américains avaient promis l'asile en Amérique au peuple hmong s'ils servaient d'informateurs. En effet, les Hmongs ont donc joué ce rôle durant cette guerre et 300000 d'entre eux ont obtenu le statut de réfugiés aux États-Unis (Moore, 1989; Thao, 1999; Thrower Timm \& Chiang, 1997). Le gouvernement américain a utilisé des entretiens enregistrés pour déterminer le statut des Hmongs. Par contre, plus d'un million de Hmongs vivent encore au Vietnam et ceux qui y habitent toujours ont subi des représailles du gouvernement vietnamien. Chez les Premières Nations du Canada, des chercheurs ont mené des groupes de discussion dans des communautés autochtones afin d'obtenir leur savoir (par exemple à propos des propriétés des plantes de la forêt). Les résultats de leurs recherches ne servent pas souvent au profit des communautés interrogées et ils leur sont rarement communiqués (Edwards, Lund, Mitchell, \& Andersson, 2008). Ainsi, ces expériences de recherche laissent ces communautés perplexes à l'égard des chercheurs (Smith 2003). Dans ce cas-ci et pour certaines populations autochtones revendiquant des droits, les groupes de discussion peuvent paraître menaçants et insécurisants. Les participants sont difficiles à recruter et ceux qui participent sont peu éloquents. Dans de tels contextes sociopolitiques, les témoignages verbaux méritent d'être complétés et approfondis par des données visuelles pour respecter et sécuriser les participants et ainsi favoriser une participation active à la fois verbale et non verbale. Dans les deux cas, les dessins ont joué le rôle d'artéfacts ayant expliqué des réalités complexes, comme la littérature l'avait indiqué (Stock et al., 2012; Swain, Kinnear, \& Steinman, 2011). Comme le précise Baribeau (2009), il est essentiel de mettre en place les conditions nécessaires préalables au bon déroulement du groupe de discussion. Lors des deux expérimentations, l'intériorisation et le silence permis par le dessin faisaient partie des conditions préalables. Il est possible de remarquer qu'en combinant les dessins avec les groupes de discussion, les participants étaient engagés, participatifs, et ils ont d'ailleurs apprécié le dessin-entretien : « Je suis très satisfait, car les images expriment mes mots et mes idées »[traduction 
libre] (Enseignant hmong. Notes de C. Lavoie, Vietnam, 29 juillet 2008). De même, certains groupes minoritaires n'osent pas parler, car ils craignent pour leur sécurité ou pour leurs droits. Pour ces raisons, le groupe de discussion, à lui seul, revêt certaines limitations envers les populations minoritaires et/ou marginalisées. Le fait de pouvoir dessiner pendant la présentation individuelle et la discussion de groupe permet un consentement silencieux, une approbation non verbale pour les participants plus timides ou pour ceux hésitant à s'exprimer dans la langue des échanges. Cette particularité par rapport au groupe de discussion offre un avantage aux minorités ethnoculturelles et aux groupes marginalisés. Ces constats confirment l'importance et la cohérence culturelle de favoriser le silence, le visuel, la narration et la discussion collective pour obtenir une discussion authentique dans des contextes spécifiques de recherche réalisée auprès des minorités ethnoculturelles (Denzin et al., 2008; Martin \& Mirraboopa, 2003; Smith, 2003; Stocek \& Mark, 2009; Stock et al., 2012).

\section{Les avantages du dessin-entretien}

L'authenticité que les participants et les chercheurs attribuent aux données n'est pas négligeable. Les participants des études présentées ont d'ailleurs affirmé que cet outil les a aidés à décrire leurs expériences personnelles. La visée théorique de fournir des données fidèles à la réalité en contexte de coconstruction de sens (Morissette, 2011) est commune au groupe de discussion et au dessin-entretien. Ainsi, le dessin-entretien, lors des deux expérimentations, a permis aux chercheuses d'approfondir leur compréhension quant à la réalité des enseignants hmongs au Vietnam et à l'expérience scolaire des persévérants universitaires des Premières Nations (Joncas, 2013; Lavoie \& Benson, 2011).

Les auteures sont d'avis que le dessin-entretien facilite le développement des connaissances concernant les minorités ethnoculturelles par le simple fait que le dessin permet d'entamer le dialogue en laissant un moment d'intériorisation, de silence et de respect culturel tout en bâtissant progressivement la confiance. Ainsi, cet outil qualitatif permet de faire ressortir des données que les méthodes plus factorielles et statistiques ne permettent pas de révéler et respecte le style de communication culturelle des minorités ethnoculturelles chez qui le silence a une grande importance. L'étape du dessin individuel dispose les participants à entamer ce que Leclerc et al. (2011) qualifient de «bonne conversation »: «Une "bonne conversation" suppose l'instauration d'un climat respectueux, mais convivial et le moins artificiel possible » (p. 151). Auprès des minorités ethnoculturelles, pour démarrer une «bonne conversation», il faut préalablement un moment d'intériorité, de 
silence. C'est pour cette raison, entre autres, que le groupe de discussion est combiné au dessin durant le dessin-entretien. Ces deux temps de dessin et de groupe de discussion comportent l'avantage de rééquilibrer la participation entre les gens ayant plus de facilité à s'exprimer verbalement et ceux ayant de la facilité à s'exprimer visuellement, car il n'est pas toujours évident pour les participants qui, par exemple, ne sont pas à l'aise avec la langue des échanges de participer au groupe de discussion. Par conséquent, à lui seul, le groupe de discussion devient limitatif pour plusieurs participants allophones. Ainsi, l'utilisation du dessin diminue les barrières de la langue souvent présentes lors des recherches auprès des minorités ethnoculturelles. Cet avantage confirme ce que Bishop (2006) et Weber (2008) avançaient en écrivant que le dessin en contexte de langue seconde facilite les échanges. En effet, la diversité des modalités de communication (verbales et non verbales) favorise la communication entre les participants de langues différentes et entre le chercheur et les participants. L'interprète d'UNICEF participant à l'expérimentation au Vietnam a d'ailleurs précisé : «L'exercice du dessin-entretien s'est déroulé dans un esprit collaboratif harmonieux » [traduction libre] (Notes de C. Lavoie, Vietnam, 29 juillet 2008).

Les auteures croient que la profondeur des données n'aurait pas été la même si l'outil de collecte de données utilisé n'avait pas débuté par un dessin. Par ailleurs, cet outil diminue le stress lié à la prise de parole. Cet avantage est soulevé par Stock et al. (2012) qui ont travaillé avec le dessin lors d'une recherche intégrant des communautés autochtones des territoires du nord de l'Australie (Îles Tiwi et Darwin). Ces auteurs ont également remarqué que le dessin demande du temps et, par le fait même, il diminue la pression engendrée par les questions ouvertes et l'anxiété que peut provoquer une entrevue formelle. Stock et al. (2012) attribuent ce fait à l'utilisation du dessin qui minimise les contacts directs des yeux, ce qui diminuerait la gêne et encouragerait l'expression sur des thématiques sensibles. En effet, lors des deux recherches présentées, les participants ne semblaient pas gênés même si parfois des passages plus difficiles étaient abordés :

Moi, je suis angoissée pour les travaux d'équipe. Quand tu es dans une nouvelle classe, tu ne connais personne, tu ne sais pas avec qui tu vas être. Tu espères que personne ne se connaisse pour qu'il $\mathrm{y}$ en ait qui soit seul et qui comprenne. Parce que des fois, tu arrives et tu es comme le pion en trop. Tu n'as pas le choix. (étudiante autochtone, $1^{\text {er }}$ février 2013, étape trois du dessinentretien : la préanalyse en groupe). 
L'atmosphère qui régnait lors des dessins-entretiens inspirait la confiance, la complicité et l'ouverture. Cette façon de procéder permet de recueillir les propos des participants plus gênés et ayant moins tendance à s'exprimer sur des questions ouvertes au groupe en général (Knowles \& Cole, 2008; Lavoie, 2011). Au final, les participants réunis ont semblé être satisfaits des rencontres et avoir apprécié ces moments de partage qui leur ont été accordés. Un étudiant a même mentionné à la suite du dessin-entretien qu'il faudrait plus de moments de parole tels que celui qui venait de se produire entre les étudiants des Premières Nations de l'Université.

De son côté, le groupe de discussion optimise la collecte d'informations que les phases de dessins ne permettent pas. L'ajout de l'aspect collectif du groupe de discussion lors des dernières phases du dessin-entretien favorise la relation entre les participants. Le groupe de discussion du dessin-entretien se distingue également des groupes de discussion traditionnels par le rôle attribué aux participants :

Traditionnellement, les entretiens en groupe focalisé se tenaient dans le cadre d'une seule rencontre, puis le chercheur repartait avec les données qu'il analysait pour les besoins de sa recherche ou de ses mandataires. En d'autres mots, il s'agissait d'un processus à sens unique. Les participants étaient sollicités comme de simples fournisseurs de données : ils n'avaient pas accès aux résultats de leurs échanges; ils n'étaient pas consultés quant à l'utilisation faite de ces résultats; et ils étaient laissés en dehors de la démarche d'analyse. (Leclerc et al., 2011, p. 146).

Dans le cas du dessin-entretien, le groupe de discussion permet une triangulation des données et une hiérarchisation des thèmes avec et par les participants. Suivant cela, le dessin-entretien respecte l'un des concepts fondamentaux des cultures autochtones, celui du savoir relationnel (Absolon \& Willet, 2004; Battiste, 2008; Smith, 2003; Stocek \& Mark, 2009). Le savoir se crée dans la relation entre les personnes selon cette conception. Le climat des échanges est bonifié grâce à ce processus cyclique entre les moments de réflexion personnelle et collective et entre le verbal et le non-verbal. De plus, le dessin-entretien, par son processus méthodologique cyclique et son utilisation $\mathrm{du}$ dessin, donne aux participants le temps dont ils ont besoin pour s'exprimer. La disposition circulaire des participants favorise les échanges (Foy, 2009; Lavallée, 2009; Styres et al., 2010), mais également la construction du sens au milieu de ce cercle (voir la Figure 3). En guise d'exemple, les auteures ont vu des participants empiler des dessins pour signaler la similitude de leurs idées, découper une partie de leur dessin et la placer au centre pour signaler une 
information nouvelle, se tenir la main en signe d'appui de leur témoignage ou encore ajouter des mots à côté de certains éléments du dessin (voir la Figure 4). À la différence du groupe de discussion, l'espace central du cercle se remplit, se construit de témoignages verbaux et non verbaux.

Finalement, le dessin-entretien a également comme avantage d'entamer une première analyse des données en collaboration avec les participants. L'aspect consensuel du dessin-entretien permet une thématisation et une triangulation des données (Lavoie \& Benson, 2011). Au final, les thèmes ressortis lors du dessin-entretien sont originaux et n'auraient souvent pas été envisagés par le chercheur. Ce processus de triangulation fait par les participants approfondit l'interprétation des données, ce qui n'est pas le cas pour des données issues de questionnaires ou des groupes de discussion traditionnels. Une autre particularité du dessin-entretien est qu'il peut être coanimé par un participant choisi par le groupe. Cette coanimation mène parfois vers de nouvelles questions. De plus, le choix par le groupe d'un animateur parmi les participants permet à celui-ci d'expliquer ou de valider l'information dans plusieurs langues auprès des participants soit en utilisant la langue minoritaire, s'il la parle, ou en permettant un temps de traduction par d'autres participants. De cette façon, le dessin-entretien a l'avantage de rééquilibrer les rapports de force entre les locuteurs de la langue dominante (chercheur et certains participants) et ceux ne maîtrisant pas parfaitement cette langue.

Le dessin-entretien semble alors être une innovation appropriée pour travailler avec des peuples minoritaires. Elle permet de surpasser les barrières du langage et de la gêne, amène une forme de transparence et d'authenticité aux données, encourage la réflexion individuelle et de groupe, permet la liberté d'expression et l'utilisation de différents médias de communication, favorise le partage d'idées et la coconstruction de la connaissance par le dialogue en groupe et mène vers une triangulation des données par les participants eux-mêmes.

\section{Les limites du dessin-entretien}

Toutefois, il est important de rappeler que cet outil comporte également quelques limites. Par exemple, lors des phases de dessin du dessin-entretien, il peut arriver que les participants manquent d'inspiration. De plus, une mauvaise dynamique de groupe peut entraîner des biais au processus du dessin-entretien, que ce soit par des conflits d'intérêts, des jugements dévalorisants entre les participants ou autres. Malgré les différentes modalités de communication, certains participants peuvent ne pas s'exprimer et d'autres peuvent monopoliser 
la parole. Également, il se peut que durant la dernière étape du dessin-entretien les participants n'arrivent pas à un commun accord sur le choix des dessins.

Il est également important de souligner que le dessin-entretien a été seulement utilisé auprès de deux groupes. Ces deux groupes réunissaient une majorité de participants de sexe féminin. Ainsi, il peut être pertinent de se questionner à savoir si l'utilisation du dessin-entretien est aussi efficace chez d'autres populations de même que chez des participants de sexe masculin. Le même questionnement peut être appliqué aux enfants, adolescents et aînés, car les participants aux études de Lavoie (2008) et Joncas (2013) étaient tous des adultes. Il serait alors important d'utiliser cet outil auprès de groupes de participants différents afin de le bonifier. Dans un autre ordre d'idées, le dessin-entretien est difficilement réalisable en contexte de grand groupe. Ainsi, l'échantillon est généralement assez petit et donc rarement généralisable.

Enfin, le dessin-entretien est un outil flexible, mais il peut mener à des difficultés d'animation. Parfois, il peut être difficile pour l'animateur de garder la concentration sur l'objet d'étude ou de suivre la discussion. En effet, la flexibilité du dessin-entretien rend aisé le changement de sujets par les participants. Ainsi, cette flexibilité est un avantage d'une certaine façon en permettant l'accès à des avenues non envisagées par le chercheur, mais, incontrôlée, elle peut mener vers une collecte de données hors sujet. Suivant cela, il est important que le chercheur connaisse bien son objet d'étude et qu'il demeure attentif aux glissements de tous genres en usant de discernement, de délicatesse et d'ouverture. Il doit aussi faire preuve de rigueur pour ne pas contaminer les données par ses propres jugements et interprétations. Rappelons que cela peut s'avérer difficile puisque le chercheur est plongé en contexte culturel différent. En effet, les normes sociales et culturelles diffèrent d'une ethnie à l'autre, ce qui peut mener vers des biais d'interprétation du chercheur qui ne possède pas la même culture que les participants.

\section{Conclusion}

En somme, les deux expérimentations du dessin-entretien présentées ont permis de faciliter une collecte de données fiables auprès de minorités ethnoculturelles, collecte qui souvent est qualifiée de laborieuse étant donné les multiples biais associer à la relation entre les participants et le chercheur. Ces expérimentations ont ainsi permis d'affirmer que l'utilisation d'un outil de collecte comportant plusieurs médias et respectant certains principes des cultures autochtones renseigne davantage le chercheur sur la réalité des participants. De cette façon, les données collectées reflètent une authenticité que les outils plus traditionnels ne permettent pas d'atteindre auprès de ces populations. Le dessin-entretien laisse plus de temps et permet l'utilisation de 
la communication verbale et non verbale pour répondre à des questions complexes. Il supporte les participants dans leur réflexion sur leur propre expérience et facilite le partage d'expériences. De plus, une spécificité de cet outil est qu'il mène à une première phase de triangulation des données par les participants au moment de la collecte. Le dessin-entretien offre une solution de rechange intéressante à l'entretien de groupe en permettant de partager et de valider les significations des participants, en les situant comme des sujets réflexifs et participant à la préanalyse.

Pour terminer, quelques recommandations concernant l'utilisation du dessin-entretien doivent être formulées. D'abord, il est important de laisser assez de temps aux participants pour faire le dessin. D'ailleurs, il est normal que le démarrage du dessin prenne quelques minutes, car les participants doivent réfléchir. Ensuite, comme il est difficile de prévoir la réaction et le degré d'engagement des participants, il est recommandé de préparer une autre forme de production visuelle. Par exemple, si les participants n'ont aucune envie de dessiner, il pourrait être intéressant de planifier le matériel nécessaire pour faire un collage plutôt qu'un dessin. Des images de magazines peuvent stimuler la réflexion et l'imagination des participants, par exemple. Enfin, lorsque chaque dessin est terminé, il est recommandé de prendre des photos des dessins individuels afin de garder des copies pour l'analyse et l'interprétation des données au cas où il faudrait découper ou modifier les dessins individuels originaux lors de la dernière étape du dessin-entretien collectif.

Sans remettre en question la pertinence de l'utilisation du dessin avec les minorités ethnoculturelles, il est intéressant de se demander si l'utilisation du dessin-entretien serait aussi avantageuse pour des études auprès de participants n'accordant pas autant d'importance aux représentations visuelles. Il serait pertinent d'explorer cette question pour constater si les dessins agissent comme artéfacts ou, au contraire, nuisent ou ralentissent la collecte de données. Également, de par son caractère multimodal, cet outil mériterait d'être expérimenté auprès des populations analphabètes.

\section{Notes}

${ }^{1}$ United Nations Children Fund.

${ }^{2}$ Ministry of Education and Training.

${ }^{3}$ United Nations Educational, Scientific and Cultural Organization.

${ }^{4}$ United Nations Girls' Education Initiative. 
${ }^{5}$ «During group discussions, some participants may be more talkative while others may be better at drawing. Each contribution is valued, however, because it leads the group further in its understanding of its reality. This means that it is important for the facilitator to leave space for silence as well as for drawing if participants want to contribute» (Lavoie \& Benson, 2011, p. 276)

\section{Références}

Absolon, K., \& Willet, C. (2004). Aboriginal research: berry picking and hunting in the $21^{\text {st }}$ century. First Peoples Child and Family Review, 1(1), 517.

Archibald, J.- A. (2008). An indigenous storywork methodology. Dans J. G. Knowles, \& L. A. Cole (Éds), Handbook of the arts in qualitative research (pp. 371-458). Thousand Oaks, CA : Sage.

Baribeau, C. (2009). Analyse des données des entretiens de groupe. Recherches qualitatives, 28(1), 133-148.

Baribeau, C., \& Germain, M. (2010). L'entretien de groupe : considérations théoriques et méthodologiques. Recherches qualitatives, 29(1), 28-49.

Barthes, R. (1981). Camera Lucida: reflections on photography. New York, NY : Hill \& Wang.

Barthes, R. (1983). A Barthes reader. New York, NY : Hill \& Wang.

Battiste, M. (2008). Research ethics for protecting indigenous knowledge and heritage : institutional and researcher responsibilities. Dans N. K. Denzin, Y. S. Lincoln, \& L. T. Smith (Éds), Handbook of critical and indigenous methodologies (pp. 497-511). Thousand Oaks, CA : Sage.

Benson, C., \& Kosonen, K. (2011). A critical comparison of language-ineducation policy and practice in four Southeast Asian countries and Ethiopia. Dans T. Skutnabb-Kangas, \& K. Heugh (Éds), Multilingual education and sustainable diversity work from periphery to centre (pp. 134-163). Londres : Routledge.

Bernhard, J. K., Cummins, J., Campoy, F. I., Ada, A. F., Winsler, A., \& Bleiker, C. (2006). Identity text and literacy development among preschool english language learners : enhancing learning opportunities for children at risk for learning disabilities. Teacher College Record, 108(11), 2380-2405.

Birch, L., \& Pétry, F. (2011). L'utilisation des groupes de discussion dans l'élaboration des politiques de santé. Recherches qualitatives, 29(3), 103132. 
Bishop, P. A. (2006). The promise of drawing as visual method in middle grades research. Middle Grades Research Journal, 1(2), 33-46.

Calderon, J. L., Baker, R. S., \& Wolf, K. E. (2000). Focus groups : a qualitative method complementing quantitative research for studying culturally diverse groups. Education for Health, 13(1), 91-95.

Collignon, F. F. (1994). From Paj Ntaub to paragraphs : perspectives in Hmong processes of composing. Dans V. John-Steiner, C. P. Panofsky, \& L. W. Smith (Éds), Sociocultural approaches to language and literacy (pp. 331-346). Melbourne, AU : Cambridge University Press.

Denzin, N. K., Lincoln, Y. S., \& Smith, L. T. (2008). Handbook of critical and indigenous methodologies. San Franscico, CA : Sage.

Edwards, K., Lund, C., Mitchell, S., \& Andersson, N. (2008). Trust the process: community-based researcher partnerships. A Journal of Aboriginal and Indigenous Community Health, 6(2), 187-199.

Ehrlen, K. (2009). Drawings as representations of children's conceptions. International Journal of Science Education, 31(1), 41-57.

Finley, S. (2008). Arts-based research. Dans J. G. Knowles, \& L. A. Cole (Éds), Handbook of the arts in qualitative research (pp. 71-80). Thousand Oaks, CA : Sage.

Fournier, S., \& Côté, P. (2008, Août). Projet de recherche sur le génocide des Tutsi au Rwanda de 1994 auprès d'enfants dont les parents sont génocidaires ou rescapés : création d'images, culture de la paix et de la réconciliation. Communication présentée au Séminaire mondial, colloque OMEP-CANADA : nourrir la paix avec les enfants, Québec, Canada.

Foy, J. (2009). Incorporating talk story into the classroom. First Nations Perspectives, 2, 23-33.

Haig-Brown, C. (2010). Indigenous thought, appropriation and non-aboriginal people. Canadian Journal of Education, 33(4), 925-950.

Halcomb, E. J., Gholizadeh, L., DiGiacomo, M., Phillips, J., \& Davidson, P. M. (2007). Literature review : considerations in undertaking focus group research with culturally and linguistically diverse groups. Journal of Clinical Nursing, 16(6), 1000-1011.

Joncas, J.- A. (2013). Apport à la compréhension de l'expérience scolaire de persévérants universitaires des Premières Nations au Québec: le cas d'étudiants de l'UQAC (Mémoire de maîtrise inédit). Université du Québec à Chicoutimi, QC. 
Kendrick, M., \& Jones, S. (2008). Girls' visual representations of literacy in a rural Ugandam community constructions of literacy. Canadian Journal of Education, 31(2), 371-404.

Kendrick, M., \& McKay, R. (2004). Drawings as an alternative way of understanding young children's constructions of literacy. Journal of Early Childhood Literacy, 4(1), 109-128.

Knowles, J. G., \& Cole, A. L. (2008). Handbook of the arts in qualitative research. Thousand Oaks, CA : Sage.

Kosonen, K. (2004). Language in education policy and practice in Vietnam. Hanoï, Vietnam : UNICEF.

Lavallée, L. F. (2009). Practical application of an indigenous research framework and two qualitative indigenous research methods: sharing circles and Anishnaabe symbol-based reflection. International Journal of Qualitative Methods, 8(1), 21-40.

Lavoie, C. (2008). Rapport de stage sur les méthodes de recherche-action. Hanoï, Vietnam : UNICEF.

Lavoie, C. (2011). The educational realities of Hmong communities in Vietnam: the voices of teachers. Critical Inquiry in Language Studies, $8(2), 153-175$.

Lavoie, C., \& Benson, C. (2011). Drawing-voice as a methodological tool for understanding teachers' concerns, in a pilot Hmong-Vietnamese bilingual education program in Vietnam. Language, Culture and Curriculum, 24(3), 269-286.

Leclerc, C., Bourassa, B., Picard, F., \& Courcy, F. (2011). Du groupe focalisé à la recherche collaborative: avantages, défis et stratégies. Recherches qualitatives, 29(3), 145-167.

Martin, K., \& Mirraboopa, B. (2003). Ways of knowing, being and doing : a theoretical framework and methods for indigenous and indigenist re-search. Journal of Australian Studies, 27(76), 203-214.

McMullen, B., \& Rohrbach, A. (2003). Distance education in remote Aboriginal communities: barriers, learning styles and best practices. Prince Georges, BC : College of New Caledonai Press.

Ministère de l'Éducation, du Loisir et du Sport. (2009, Septembre). L'éducation des populations scolaires dans les communautés autochtones du Québec. Bulletin statistique de l'éducation, 39. 
Moore, D. L. (1989). Dark sky, dark land: stories of the Hmong boy scouts of troop 100. Eden Prairie, MN : Tessera.

Morrissette, J. (2011). Ouvrir la boîte noire de l'entretien de groupe. Recherches qualitatives, 29(3), 7-32.

Överlien, C., Aronsson, K., \& Hydén, M. (2005). The focus group interview as an in-depth method? Young women talking about sexuality. International Journal of Social Research Methodology, 8(4), 331-344.

Prasad, G. (2010, Mars). Alter(n)ative research practices: methodological considerations for working with children as co-researchers. Communication présentée à la Conférence Comparative international education society. Chicago : University of Chicago.

Restoule, J.- P., Archibald, J.- A., Lester-Smith, D., Parent, A., \& Smilie, C. A. (2010). Connecting to spirit in indigenous research. Canadian Journal of Native Education, 33(1), 1-11.

Rudduck, J., \& Hopkins, D. (1985). Research as a basis for teaching : readings from the work of Lawrence Stenhouse. London, ON: Heinemann Educational Books.

Salem, R. A. (2000). Witness to genocide : the children of Rwanda. New York, NY : Friendship Press-National Council of the churches of Christ in the USA.

Shea, P. D., \& Yang, Y. (1995). The whispering cloth. Honesdale, PN : Boyds Mills.

Smith, L. T. (2003). Decolonizing methodologies: research and indigenous peoples. New York, NY : Zed books.

Stocek, C., \& Mark, R. (2009). Indigenous research and decolonizing methodologies : possibilities and opportunities. Dans J. Langdon (Éd.), Indigenous knowledges, development and education (pp. 73-96). Rotterdam, NL : Sense Publishers.

Stock, C., Mares, S., \& Robinson, G. (2012). Telling and re-telling stories : the use of narrative and drawing in a group intervention with parents and children in a remote aboriginal community. The Australian and New Zealand Journal of Family Therapy, 33(2), 157-170.

Styres, S., Zinga, D., Bennett, S., \& Bomberry, M. (2010). Walking in two worlds : engaging the space between indigenous community and academia. Canadian Journal of Education, 33(3), 617-648. 
Swain, M., Kinnear, P., \& Steinman, L. (2011). Sociocultural theory in second language education: an introduction through narratives. Bristol, UK: Multilingual matters textbooks.

Thao, P. (1999). Mong education at the crossroads. Lanham, MD : University Press of America.

Thrower Timm, J., \& Chiang, B. (1997). Hmong culture and cognitive style. Dans B. J. Robinson Shade (Éd.), Culture, style and the educative process (pp. 105-117). Springfield, IL : Charles C. Thomas.

UNICEF, MOET, UNESCO, \& UNGEI. (2008). The transition of ethnic minority girls from primary to secondary education. Hanoï, Vietnam : UNICEF.

Wang, C., \& Burris, M. (1997). Photovoice : concept, methodology, and use for participatory needs assessment. Health Education and Behavior, 24(3), 369-387.

Weber, S. (2008). Visual images in research. Dans J. G. Knowles, \& A. L. Cole (Éds), Handbook of the arts in qualitative research (pp. 41-50). Thousand Oaks, CA : Sage.

Weber-Pillwax, C. (1999). Indigenous research methodology: exploratory discussion of an elusive subject. Journal of Educational Thought, 33(1), 14.

Wilson, S. (2003). Progressing toward an indigenous research paradigm in Canada and Australia. Canadian Journal of Native Education, 27(2), 161178.

Constance Lavoie occupe un poste de professeure en didactique du français au primaire à l'Université du Québec à Chicoutimi. En 2009, elle a obtenu son doctorat en didactique des langues secondes à l'Université McGill. Ses champs d'intérêt en recherche portent sur l'enseignement de la didactique de l'oral auprès des communautés culturelles et linguistiques minoritaires.

Jo-Anni Joncas a terminé en 2013 une maîtrise en éducation à l'Université du Québec à Chicoutimi et est candidate au doctorat en administration et évaluation en éducation à l'Université Laval. Ses champs de recherche concernent la sociologie de l'éducation, les études autochtones et l'enseignement supérieur. Elle s'intéresse également aux méthodes de recherche qualitatives. 